Document downloaded from:

http://hdl.handle.net/10251/60383

This paper must be cited as:

Albanese, AA.; Bonet Solves, JA.; Ricker, WJ. (2014). Characterizing Fréchet-Schwartz spaces via power bounded operators. Studia Mathematica. 224(1):25-45. doi:10.4064/sm224-1-2

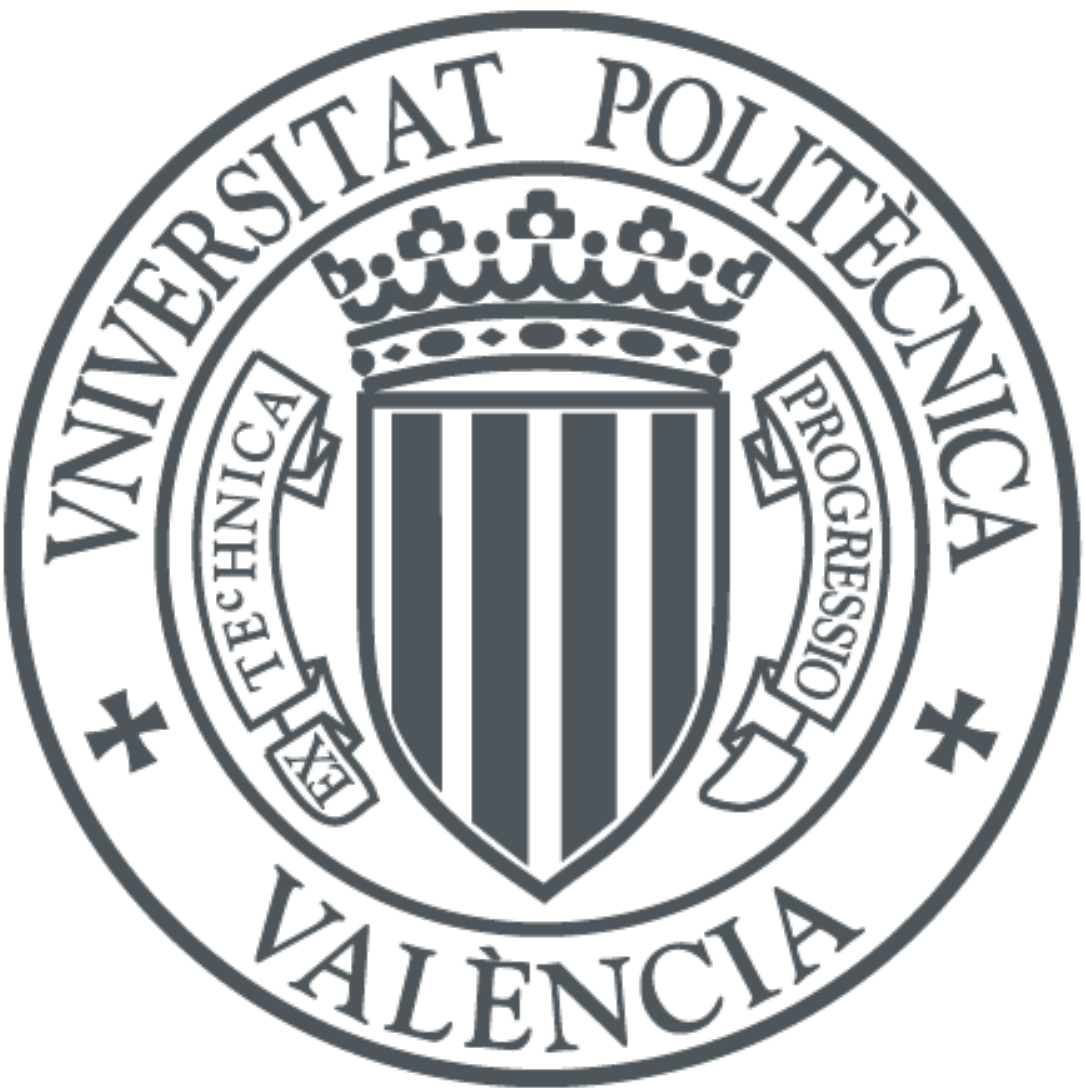

The final publication is available at

http://dx.doi.org/10.4064/sm224-1-2

Copyright Polskiej Akademii Nauk, Instytut Matematyczny (Polish Academy of Sciences, Institute of Mathematics)

Additional Information 


\title{
CHARACTERIZING FRÉCHET-SCHWARTZ SPACES VIA POWER BOUNDED OPERATORS
}

\author{
ANGELA A. ALBANESE, JOSÉ BONET AND WERNER J. RICKER
}

\begin{abstract}
We characterize Köthe echelon spaces (and, more generally, those Fréchet spaces with an unconditional basis) which are Schwartz, in terms of the convergence of the Cesàro means of power bounded operators defined on them. This complements similar known characterizations of reflexive and of FréchetMontel spaces with a basis. Every strongly convergent sequence of continuous linear operators on a Fréchet-Schwartz space does so in a special way. We single out this type of "rapid convergence" for a sequence of operators and study its relationship to the structure of the underlying space. Its relevance for Schauder decompositions and the connection to mean ergodic operators on Fréchet-Schwartz spaces is also investigated.
\end{abstract}

\section{INTRODUCTION}

A continuous linear operator $T$ acting in a Fréchet space $X$ is called power bounded (resp. mean ergodic, resp. uniformly mean ergodic) if the sequence $\left\{T^{n}\right\}_{n=1}^{\infty}$ of iterates (resp. the sequence of the Cesàro means $\left\{\frac{1}{n} \sum_{k=1}^{n} T^{k}\right\}_{n=1}^{\infty}$ ) is equicontinuous (resp. convergent for the strong operator toplogy $\tau_{s}$, resp. convergent for the uniform operator topology $\tau_{b}$ ). J. von Neumann (1931) proved that unitary operators in Hilbert spaces are mean ergodic. F. Riesz (1938) showed that every power bounded operator in an $L^{p}$-space $(1<p<\infty)$ is mean ergodic. In 1939 E.R. Lorch extended this result to all reflexive Banach spaces. It quickly became evident that there was an intimate connection between geometric properties of the underlying Banach space $X$ and mean ergodic operators on $X$. Concerning the converse, in 1997 E.Yu.Emel'yanov showed that every Banach lattice with the property that every power bounded operator on the space is mean ergodic is necessarily reflexive, [13]. A major breakthrough came in 2001 when V.P. Fonf, M. Lin and P. Wojtaszczyk, [14], established the following characterizations for a Banach space $X$ with a basis:

(i) $X$ is finite-dimensional if and only if every power bounded operator on $X$ is uniformly mean ergodic.

(ii) $X$ is reflexive if and only if every power bounded operator on $X$ is mean ergodic.

This paper initiated an immediate interest for analogous questions in the setting of Fréchet spaces.

Key words and phrases. Power bounded operator, mean ergodic operator, Fréchet-Schwartz space, Köthe echelon space, Schauder decomposition, rapid convergence.

Mathematics Subject Classification 2010: Primary: 46A04; Secondary: 46A11, 46A45, 47A35, $47 \mathrm{~B} 37$. 
The result of Emel'yanov was extended in [10], where it was shown that a Fréchet lattice $X$ is reflexive if and only if every power bounded operator on $X$ is mean ergodic. An analogue of (i) is also presented in [10]. Namely, a discrete Fréchet lattice $X$ is Montel (i.e., bounded sets are relatively compact) if and only if every power bounded operator lying in the centre $Z(X)$ of $X$ is uniformly mean ergodic. Concerning further results along the lines of (i) and (ii) above, it is known that a Fréchet space $X$ with a basis is Montel if and only if every power bounded operator on $X$ is uniformly mean ergodic, [1, Theorem 1.3], and that $X$ is reflexive if and only if every power bounded operator on $X$ is mean ergodic, [1, Theorem 1.4]. For analogous results in the setting of locally convex spaces we refer to [2]; see also [24], [25]. If the Fréchet space $X$ is not assumed to have a basis, then $X$ is Montel if and only if every power bounded, mean ergodic operator defined on any closed subspace of $X$ is uniformly mean ergodic, [1, Theorem 5.4], and $X$ is reflexive if and only if every power bounded operator defined on any closed subspace of $X$ is mean ergodic, [1, Proposition 5.1].

In a conference in Trier (Germany) in 2008, where the above mentioned results were presented for the first time, Prof. A. Pelczyński suggested that there should be similar criteria available which characterize Fréchet-Schwartz spaces. In order to be able to distinguish between "Montel and Schwartz" it is necessary to find an appropriate (and stronger) notion of operator convergence than $\tau_{b}$-convergence. The aim of this paper is to present such a notion and to invoke it to address Pelczynski's suggestion.

Let $\left\{S_{k}\right\}_{k=1}^{\infty}$ be a sequence of continuous linear operators on a Fréchet space $X$, whose topology is generated by a fundamental, increasing sequence of seminorms $\left\{q_{n}\right\}_{n=1}^{\infty}$. Then $\left\{S_{k}\right\}_{k=1}^{\infty}$ is called rapidly convergent if there exists a continuous linear operator $S$ on $X$ such that, for every $n \in \mathbb{N}$ there exists $m>n$ with

$$
\lim _{k \rightarrow \infty} \sup \left\{q_{n}\left(\left(S_{k}-S\right) x\right): q_{m}(x) \leq 1\right\}=0,
$$

in which case we write $S_{k} \stackrel{(R)}{\rightarrow} S$ for $k \rightarrow \infty$. Whenever $S_{k} \stackrel{(R)}{\rightarrow} S$ for $k \rightarrow \infty$, then also $\tau_{b}-\lim _{k \rightarrow \infty} S_{k}=S$. However, since there exist Fréchet-Montel spaces which fail to be Schwartz, $\tau_{b}$-convergence of a sequence of operators need not imply its rapid convergence: this follows from the fact that $\tau_{s^{-}}$-convergence of a sequence of operators in a Montel space implies its $\tau_{b}$-convergence and from the characterization presented in Corollary 3.4. Namely, a Fréchet space $X \neq\{0\}$ is Schwartz if and only if every sequence of operators in $X$ which is $\tau_{s}$-convergent is also rapidly convergent.

An adequate response to Pelczyński's suggestion, via the notion of rapid convergence, is presented in the final two sections. In Section 4 we introduce the new notion of an operator being rapidly mean ergodic. A deep result of S.F. Bellenot, [3], stating that each Fréchet-Schwartz space is a closed subspace of a Fréchet-Schwartz space with an unconditional basis, plays a role in establishing the main result of this section; see Theorem 4.6. Namely, let $X$ be a Fréchet space which is a closed subspace of a Fréchet space with an unconditional basis. Then $X$ is Schwartz if and only if every closed subspace $Y$ of $X$ has the property that every power bounded operator on $Y$ is rapidly mean ergodic. In the final Section 5 this result is refined (see Theorem 5.6) for the important class of Fréchet spaces $\lambda_{p}(A), p \in[1, \infty) \cup\{0\}$, known as Köthe echelon spaces, all of which have 
an unconditional basis. Indeed, it is shown that $\lambda_{p}(A)$ is Schwartz if and only if every power bounded operator on $\lambda_{p}(A)$ is rapidly mean ergodic.

\section{Preliminaries}

Our notation for locally convex Hausdroff spaces, briefly lcHs', is standard; we refer to [17], [18], [20], [22], [26]. More detailed information on Fréchet and Köthe echelon spaces can be found in [6], [7], [22]. A standard reference for mean ergodic operators is [19]. We begin with some definitions and notation which will facilitate the reading of the paper.

Let $X$ be a lcHs and $\Gamma_{X}$ a system of continuous seminorms determining the topology of $X$. The strong operator topology $\tau_{s}$ in the space $\mathcal{L}(X)$ of all continuous linear operators from $X$ into itself is determined by the family of seminorms

$$
q_{x}(S):=q(S x), \quad S \in \mathcal{L}(X),
$$

for each $x \in X$ and $q \in \Gamma_{X}$, in which case we write $\mathcal{L}_{s}(X)$. Denote by $\mathcal{B}(X)$ the collection of all bounded subsets of $X$. The topology $\tau_{b}$ of uniform convergence on bounded sets is defined in $\mathcal{L}(X)$ via the seminorms

$$
q_{B}(S):=\sup _{x \in B} q(S x), \quad S \in \mathcal{L}(X),
$$

for each $B \in \mathcal{B}(X)$ and $q \in \Gamma_{X}$; in this case we write $\mathcal{L}_{b}(X)$. For $X$ a Banach space, $\tau_{b}$ is the operator norm topology in $\mathcal{L}(X)$. If $X$ is metrizable and complete, then $X$ is called a Fréchet space. In this case $\Gamma_{X}$ can be taken countable. The identity operator on a lcHs $X$ is denoted by $I$. Of course, for $T \in \mathcal{L}(X)$ we define $\operatorname{Ker} T:=T^{-1}(\{0\})$.

By $X_{\sigma}$ we denote $X$ equipped with its weak topology $\sigma\left(X, X^{\prime}\right)$, where $X^{\prime}$ is the topological dual space of $X$, and $X_{\sigma}^{\prime}$ denotes $X^{\prime}$ equipped with its weak-star topology $\sigma\left(X^{\prime}, X\right)$. Given $T \in \mathcal{L}(X)$, its dual operator $T^{\prime}: X^{\prime} \rightarrow X^{\prime}$ is defined by $\left\langle x, T^{\prime} x^{\prime}\right\rangle=\left\langle T x, x^{\prime}\right\rangle$ for all $x \in X, x^{\prime} \in X^{\prime}$. Note that $T^{\prime} \in \mathcal{L}\left(X_{\sigma}^{\prime}\right)$, [18, p.134].

Recall that a Fréchet space $X$ with a basis of decreasing, absolutely convex 0-neighbourhoods $\left\{U_{n}\right\}_{n=1}^{\infty}$ is Schwartz if

$$
\forall n \in \mathbb{N} \exists m>n \forall \varepsilon>0 \exists F_{\varepsilon} \subseteq X \text { finite such that } U_{m} \subseteq F_{\varepsilon}+\varepsilon U_{n},
$$

or, equivalently, if

$\forall n \in \mathbb{N} \exists m>n \forall \varepsilon>0 \exists L_{\varepsilon} \subseteq X$ relatively compact such that $U_{m} \subseteq L_{\varepsilon}+\varepsilon U_{n}$,

[15, p.276]. Therefore, a Fréchet space $X$ is Schwartz if and only if $X$ can be written as a projective limit via continuous linear linking operators $S_{n}: X_{n+1} \rightarrow$ $X_{n}$, for $n \in \mathbb{N}$, with each $X_{n}$ a Banach space, such that, for every $n \in \mathbb{N}$, there exists $m>n$ with $\left(S_{m-1} \circ \ldots \circ S_{n}\right): X_{m} \rightarrow X_{n}$ a compact operator. Further details about Schwartz lcHs' can be found in [15], [20], [22].

The following characterization of Fréchet-Schwartz spaces, due to Bonet, Lindström, Valdivia, [9], is a version of the Josefson-Nissenzweig theorem for Fréchet spaces. See also [8], [21].

Theorem 2.1. A Fréchet space $X$ is Schwartz if and only if for each sequence $\left\{x_{k}^{\prime}\right\}_{k=1}^{\infty} \subseteq X^{\prime}$ which satisfies $\lim _{k \rightarrow \infty} x_{k}^{\prime}=0$ in $X_{\sigma}^{\prime}$, there exists $n \in \mathbb{N}$ such that $\left(\sup _{x \in U_{n}}\left|\left\langle x, x_{k}^{\prime}\right\rangle\right|\right)_{k=1}^{\infty} \in c_{0}$. 
A Schauder decomposition of a Fréchet space $X$ is a sequence $\left\{P_{j}\right\}_{j=1}^{\infty} \subseteq \mathcal{L}(X)$ of operators in $X$ satisfying the following properties:

(S1) $P_{i} P_{j}=P_{\min \{i, j\}}$, for all $i, j \in \mathbb{N}$,

(S2) $\tau_{s}-\lim _{j \rightarrow \infty} P_{j}=I$, and

(S3) $P_{i} \neq P_{j}$ if $i \neq j$;

see [11], [16]. According to (S1) each $P_{j}$, for $j \in \mathbb{N}$, is a projection. Condition (S2) implies that $\left\{P_{j}\right\}_{j=1}^{\infty}$ is an equicontinuous subset of $\mathcal{L}(X)$. If the range $P_{j}(X)$ is a finite-dimensional space for each $j \in \mathbb{N}$, then $\left\{P_{j}\right\}_{j=1}^{\infty}$ is said to be a finite-dimensional Schauder decomposition of $X$.

By setting $Q_{1}:=P_{1}$ and $Q_{n}:=P_{n}-P_{n-1}$ for $n \geq 2$ we arrive at a sequence of pairwise orthogonal projections (i.e., $Q_{n} Q_{m}=0$ if $n \neq m$ ). Moreover, (S2) implies that $\sum_{n=1}^{\infty} Q_{n}=I$, with the series converging in $\mathcal{L}_{s}(X)$, and (S3) ensures that $Q_{n} \neq 0$ for each $n \in \mathbb{N}$. If the series $\sum_{n=1}^{\infty} Q_{n}=I$ is unconditionally convergent in $\mathcal{L}_{s}(X)$, then $\left\{P_{j}\right\}_{j=1}^{\infty}$ is called an unconditional Schauder decomposition of $X$. The dual projections $\left\{P_{j}^{\prime}\right\}_{j=1}^{\infty} \subseteq \mathcal{L}\left(X_{\sigma}^{\prime}\right)$ always form a Schauder decomposition of $X_{\sigma}^{\prime},[16$, p.378].

Inspired by the work of Benndorf, [5], Díaz introduced in [12] the following notion: a Schauder decomposition $\left\{P_{j}\right\}_{j=1}^{\infty}$ satisfies the property $(S)$ if

$$
\forall n \in \mathbb{N} \exists m>n \text { with } \lim _{j \rightarrow \infty} \sup \left\{q_{n}\left(\left(I-P_{j}\right) x\right): q_{m}(x) \leq 1\right\}=0,
$$

with $\left\{q_{n}\right\}_{n=1}^{\infty}$ a fundamental, increasing sequence of continuous seminorms on $X$.

Let $X$ be a Fréchet space. A sequence $\left\{e_{i}\right\}_{i=1}^{\infty} \subseteq X$ is called a basis for $X$ if for every $x \in X$ there is a unique sequence $\left(\alpha_{i}\right)_{i=1}^{\infty}$ of scalars such that $x=\sum_{i=1}^{\infty} \alpha_{i} x_{i}$. By setting $\left\langle x, e_{i}^{\prime}\right\rangle:=\alpha_{i}$ we obtain a linear form $e_{i}^{\prime}: X \rightarrow \mathbb{C}$, necessarily continuous, which is called the $n$-th coefficient functional associated to $\left\{e_{i}\right\}_{i=1}^{\infty}$. The functionals $e_{i}^{\prime}, i \in \mathbb{N}$, are uniquely determined by $\left\{e_{i}\right\}_{i=1}^{\infty}$ and $\left\{\left(e_{i}, e_{i}^{\prime}\right)\right\}_{i=1}^{\infty}$ is a biorthogonal sequence (i.e. $\left\langle e_{i}, e_{j}^{\prime}\right\rangle=\delta_{i j}$ for $i, j \in \mathbb{N}$ ). The sequence $\left\{e_{i}^{\prime}\right\}_{i=1}^{\infty} \subseteq X^{\prime}$ is called the dual basis of $\left\{e_{i}\right\}_{i=1}^{\infty}$.

If $\left\{e_{i}\right\}_{i=1}^{\infty} \subseteq X$ is a basis for a Fréchet space $X$, then the projections $P^{(j)} \in$ $\mathcal{L}(X)$, for $j \in \mathbb{N}$, defined by

$$
P^{(j)} x:=\sum_{i=1}^{j}\left\langle x, e_{i}^{\prime}\right\rangle e_{i}, \quad x \in X,
$$

form a finite-dimensional Schauder decomposition of $X\left(\right.$ with $P^{(j)}(X)=\operatorname{span}\left\{e_{i}\right\}_{i=1}^{j}$, for $j \in \mathbb{N})$.

We conclude this section with a fact which is surely known. Since we could not find a reference, a proof is included.

Lemma 2.2. Let $E$ be a finite-dimensional lcHs and $U$ be a closed, absolutely convex 0-neighbourhood in $E$. Then there exist a subspace $F$ of $E$ with $F \subseteq U$ and a bounded (hence, relatively compact) subset $B \subseteq E$ such that $U \subseteq F+B$.

Proof. Define $F:=\cap_{\alpha>0} \alpha U$. It is routine to verify that $F$ is the largest subspace of $E$ which is contained in $U$. Of course, it may happen that $F=\{0\}$, eg. if $U$ is bounded. Since $E$ is finite-dimensional and $F$ is the largest subspace of $E$ lying in $U$, we can write $E=F \oplus G$ with $G$ a subspace of $X$ such that $B:=(2 U) \cap G$ does not contain any non-zero subspace of $E$. The claim is that $B$ is bounded (hence, 
relatively compact). Indeed, as $B$ is a closed, absolutely convex 0-neighbourhood of the finite dimensional space $G$, we may (and will) assume that $E$ is normed via a norm $\|$.$\| . Suppose that B$ is unbounded in $E$. Then there is a sequence $\left\{w_{j}\right\}_{j=1}^{\infty}$ in $B$ such that $1<\left\|w_{j}\right\|$, for all $j$, with $\left\{\left\|w_{j}\right\|\right\}_{j=1}^{\infty}$ increasing to $\infty$ as $j \rightarrow \infty$. Set $u_{j}:=w_{j} /\left\|w_{j}\right\|$, for $j \in \mathbb{N}$. Since $B$ is absolutely convex, $u_{j} \in B$ for each $j \in \mathbb{N}$. As the unit sphere of $G$ is compact, there exists $u \in G$, with $\|u\|=1$, which is the limit of some subsequence of $\left\{u_{j}\right\}_{j=1}$. Denote this subsequence in the same way. Suppose, for the moment, that $\lambda u \in B$ whenever $\lambda>1$. Since $B$ is absolutely convex, it would follow that the linear span of $\{u\}$ (a non-trivial subspace) is contained in $B$; a contradiction! So, fix $\lambda>1$. Select $j_{0} \in \mathbb{N}$ such that $\lambda<\left\|w_{j}\right\|$ for all $j>j_{0}$. Thus, $\lambda u_{j}=\left(\lambda /\left\|w_{j}\right\|\right) w_{j} \in B$ for each $j>j_{0}$. On the other hand, $\lambda u_{j} \rightarrow \lambda u$ as $j \rightarrow \infty$. Therefore $\lambda u$ belongs to the closed set $B$. This proves the claim.

It remains to show that $U \subseteq F+B$. Let $x \in U$. Then $x=f+g$ with $f \in F$, $g \in G$ and so $g=x-f \in U-U \subseteq 2 U$. Hence, $g \in B$ which shows that $x \in F+B$.

\section{Rapid CONVERgence of operators on Fréchet-SchwartZ sPaces}

Throughout this section, $X$ is a Fréchet space with a fundamental, increasing sequence of seminorms $\Gamma_{X}=\left\{q_{n}\right\}_{n=1}^{\infty}$. For each $n \in \mathbb{N}$, set $U_{n}:=\{x \in$ $\left.X: q_{n}(x) \leq 1\right\}$.

A sequence of operators $\left\{S_{k}\right\}_{k=1}^{\infty} \subseteq \mathcal{L}(X)$ is said to be rapidly convergent to $S \in \mathcal{L}(X)$ if

$$
\forall n \in \mathbb{N} \exists m>n \text { with } \lim _{k \rightarrow \infty} \sup _{x \in U_{m}} q_{n}\left(\left(S_{k}-S\right) x\right)=0 .
$$

In such a case we write $S_{k} \stackrel{(R)}{\rightarrow} S$ in $\mathcal{L}(X)$ as $k \rightarrow \infty$ or $(R)-\lim _{k \rightarrow \infty} S_{k}=S$. It is routine to verify that $S_{k} \stackrel{(R)}{\rightarrow} S$ in $\mathcal{L}(X)$ as $k \rightarrow \infty$ if and only if $\forall n \in \mathbb{N} \exists m>n \exists \alpha=\left(\alpha_{k}\right)_{k} \in c_{0}$ with $q_{n}\left(\left(S_{k}-S\right) x\right) \leq \alpha_{k} q_{m}(x) \forall x \in X$.

Clearly, $S_{k} \stackrel{(R)}{\rightarrow} S$ as $k \rightarrow \infty$ if and only if $\left(S_{k}-S\right) \stackrel{(R)}{\rightarrow} 0$ as $k \rightarrow \infty$.

Remark 3.1. If $\left\{S_{k}\right\}_{k=1}^{\infty} \subseteq \mathcal{L}(X)$ satisfies $S_{k} \stackrel{(R)}{\rightarrow} S$ for $k \rightarrow \infty$, then it is routine to check that necessarily $\tau_{b}-\lim _{k \rightarrow \infty} S_{k}=S$. Hence, also $\tau_{s}-\lim _{k \rightarrow \infty} S_{k}=S$. In particular, the limit of a rapidly convergent sequence in $\mathcal{L}(X)$ is unique.

Rapidly convergent sequences of operators are easy to exhibit.

Example 3.2. Let $X$ be a Fréchet space which is the product of a sequence of Fréchet spaces, i.e., $X=\prod_{i=1}^{\infty} X_{i}$ with each $X_{i}$ a Fréchet space. For each $i \in \mathbb{N}$, let $\Gamma_{X_{i}}=\left\{q_{n}^{(i)}\right\}_{n=1}^{\infty}$ be a fundamental, increasing sequence of seminorms for $X_{i}$. Then the sequence of seminorms given by

$$
p_{n}(x):=\sum_{i=1}^{n} q_{n}^{(i)}\left(x_{i}\right), \quad x=\left(x_{i}\right)_{i} \in X,
$$

for each $n \in \mathbb{N}$, defines the lc-topology of $X$.

Consider the sequence of operators $\left\{P^{(k)}\right\}_{k=1}^{\infty} \subseteq \mathcal{L}(X)$, where

$$
P^{(k)} x:=\left(x_{1}, \ldots, x_{k}, 0,0, \ldots\right), \quad x=\left(x_{i}\right)_{i} \in X,
$$


i.e., $P^{(k)}$ is the projection of $X$ onto $\prod_{i=1}^{k} X_{i}$. Then $(R)-\lim _{k \rightarrow \infty} P^{(k)}=I$. Indeed, for any fixed $n \in \mathbb{N}$, we have $p_{n}\left(\left(I-P^{(k)}\right) x\right)=0$ for all $x \in X$ and $k>n$.

We proceed to analyze the relationship between the rapid convergence of sequences of operators on a Fréchet space $X$ and the fact that $X$ is Schwartz.

Proposition 3.3. Let $X$ be a Fréchet-Schwartz space. If $\left\{S_{k}\right\}_{k=1}^{\infty} \subseteq \mathcal{L}(X)$ satisfies $\tau_{s}-\lim _{k \rightarrow \infty} S_{k}=S$ for some $S \in \mathcal{L}(X)$, then also $(R)-\lim _{k \rightarrow \infty} S_{k}=S$.

Proof. It suffices to consider the case when $S=0$. Since $\left\{S_{k}\right\}_{k=1}^{\infty}$ converges to 0 in $\mathcal{L}_{s}(X)$ and $X$ is Fréchet, $\left\{S_{k}\right\}_{k=1}^{\infty}$ is equicontinuous in $\mathcal{L}(X)$. Let $n \in \mathbb{N}$. Then there exists $r(n)>n$ such that

$$
S_{k}\left(U_{r(n)}\right) \subseteq U_{n}, \quad k \in \mathbb{N} .
$$

As $X$ is Schwartz, (2.1) implies that there exists $m>r(n)$ satisfying

$$
\forall \varepsilon>0 \exists x_{1}, \ldots, x_{p(\varepsilon)} \in X \text { such that } U_{m} \subseteq \cup_{i=1}^{p(\varepsilon)}\left(x_{i}+\frac{\varepsilon}{2} U_{r(n)}\right) .
$$

To prove that $(R)-\lim _{k \rightarrow \infty} S_{k}=0$ we need to show that

$$
\lim _{k \rightarrow \infty} \sup _{x \in U_{m}} q_{n}\left(S_{k} x\right)=0 .
$$

To verify (3.5), let $\varepsilon>0$ and choose $x_{1}, \ldots, x_{p(\varepsilon)} \in X$ according to (3.4). For each $i=1, \ldots, p(\varepsilon)$ we have $S_{k} x_{i} \rightarrow 0$ in $X$ as $k \rightarrow \infty$. Hence, there exists $k_{0} \in \mathbb{N}$ such that

$$
S_{k} x_{i} \in \frac{\varepsilon}{2} U_{n}, \quad k \geq k_{0}, i=1, \ldots, p(\varepsilon) .
$$

Let $x \in U_{m}$. By (3.4) there exists some $j \in\{1, \ldots, p(\varepsilon)\}$ such that $x \in x_{j}+\frac{\varepsilon}{2} U_{r(n)}$. So, for every $k \geq k_{0}$ we deduce, via (3.3) and (3.6), that

$$
S_{k} x \in S_{k} x_{j}+\frac{\varepsilon}{2} S_{k}\left(U_{r(n)}\right) \subseteq \frac{\varepsilon}{2} U_{n}+\frac{\varepsilon}{2} U_{n}=\varepsilon U_{n} .
$$

That is, $\sup _{x \in U_{m}} q_{n}\left(S_{k} x\right) \leq \varepsilon$ for all $k \geq k_{0}$. This verifies (3.5) and completes the proof.

An immediate application is the following result.

Corollary 3.4. A Fréchet space $X \neq\{0\}$ is Schwartz if and only if every $\tau_{s}$ convergent sequence in $\mathcal{L}(X)$ is also rapidly convergent in $\mathcal{L}(X)$.

Proof. The necessity of the condition is clear from Proposition 3.3.

Conversely, assume that the stated condition holds. Fix a sequence $\left\{x_{k}^{\prime}\right\}_{k=1}^{\infty} \subseteq$ $X^{\prime}$ satisfying $\lim _{k \rightarrow \infty} x_{k}^{\prime}=0$ in $X_{\sigma}^{\prime}$. Select any $a \in X \backslash\{0\}$ and choose $n \in$ $\mathbb{N}$ such that $q_{n}(a)>0$. Define now a sequence $\left\{S_{k}\right\}_{k=1}^{\infty} \subseteq \mathcal{L}(X)$ by setting $S_{k} x:=\left\langle x, x_{k}^{\prime}\right\rangle a$, for $x \in X$ and $k \in \mathbb{N}$. Clearly, $\tau_{s}-\lim _{k \rightarrow \infty} S_{k}=0$. By assumption also $(R)-\lim _{k \rightarrow \infty} S_{k}=0$ and hence, via (3.1), there is $m>n$ such that $\left(\sup _{x \in U_{m}} q_{n}\left(S_{k} x\right)\right)_{k} \in c_{0}$. But, $\sup _{x \in U_{m}} q_{n}\left(S_{k} x\right)=q_{n}(a) \sup _{x \in U_{m}}\left|\left\langle x, x_{k}^{\prime}\right\rangle\right|$, for each $k \in \mathbb{N}$, with $q_{n}(a) \neq 0$, from which it follows that $\left(\sup _{x \in U_{m}}\left|\left\langle x, x_{k}^{\prime}\right\rangle\right|\right)_{k} \in c_{0}$. So, $X$ is Schwartz by Theorem 2.1.

Note that a Schauder decomposition $\left\{P_{j}\right\}_{j=1}^{\infty}$ of $X$ satisfies the property $(S)$ if and only if $P_{j} \stackrel{(R)}{\rightarrow} I$ in $\mathcal{L}(X)$ for $j \rightarrow \infty$; see (2.3) and (3.1).

The following result is essentially Lemma 1 of [5]. 
Proposition 3.5. Let $X$ be a Fréchet-Schwartz space and $\left\{P_{j}\right\}_{j=1}^{\infty} \subseteq \mathcal{L}(X)$ be any Schauder decomposition of $X$. Then each closed subspace $P_{j}(X)$ of $X$ is Schwartz, $j \in \mathbb{N}$, and $(R)-\lim _{j \rightarrow \infty} P_{j}=I$.

Proof. Every closed subspace of a Fréchet-Schwartz space is also a Fréchet-Schwartz space, $\left[15, \S 15\right.$, Proposition 6]. So, $P_{j}(X)$ is a Fréchet-Schwartz space, for each $j \in \mathbb{N}$. Moreover, by (S3) we have that $P_{j} \rightarrow I$ in $\mathcal{L}_{s}(X)$ as $j \rightarrow \infty$ and so Proposition 3.3 implies that $(R)-\lim _{j \rightarrow \infty} P_{j}=I$.

The converse of Proposition 3.5 holds for certain kinds of Schauder decomposition (cf. the next result) but, not in general; see Example 5.1.

Proposition 3.6. Let $X$ be a Fréchet space and $\left\{P_{j}\right\}_{j=1}^{\infty} \subseteq \mathcal{L}(X)$ be a finitedimensional Schauder decomposition. If $(R)-\lim _{j \rightarrow \infty} P_{j}=I$, then $X$ is Schwartz.

Proof. Since $\left\{P_{j}\right\}_{j=1}^{\infty}$ is equicontinuous, we can select a basis of decreasing, absolutely convex 0-neighbourhoods $\left\{U_{n}\right\}_{n=1}^{\infty}$ of $X$ such that $P_{j}\left(U_{n}\right) \subseteq U_{n}$ for each $j, n \in \mathbb{N}$. To show that $X$ is Schwartz, by (2.2) it suffices to verify that

$$
\forall n \in \mathbb{N} \exists m>n \forall \varepsilon>0 \exists L_{\varepsilon} \subseteq X \text { relatively compact with } U_{m} \subseteq L_{\varepsilon}+\varepsilon U_{n} .
$$

So, fix $n \in \mathbb{N}$. Since $P_{j} \stackrel{(R)}{\rightarrow} I$ for $j \rightarrow \infty$, by (3.1) there exists $m>n$ such that $\lim _{j \rightarrow \infty} \sup _{x \in U_{m}} q_{n}\left(\left(I-P_{j}\right) x\right)=0$. Hence, given any $\varepsilon>0$, there exists $j_{0} \in \mathbb{N}$ such that $\sup _{x \in U_{m}} q_{n}\left(\left(I-P_{j_{0}}\right) x\right)<\varepsilon / 2$. It follows that

$$
x-P_{j_{0}} x=\left(I-P_{j_{0}}\right) x \in \frac{\varepsilon}{2} U_{n}, \quad x \in U_{m} .
$$

Applying Lemma 2.2 with $E:=P_{j_{0}}(X)$ and $U:=U_{m} \cap E$ we can conclude that there exists a finite-dimensional subspace $F$ of $P_{j_{0}}(X)$ contained in $U_{m}$ and a relatively compact subset $B$ of $P_{j_{0}}(X)$ such that

$$
U_{m} \cap P_{j_{0}}(X) \subseteq F+B .
$$

Since $F \subseteq U_{m}$ and $F$ is a subspace, we have $\frac{2}{\varepsilon} F=F \subseteq U_{m}$, i.e., $F \subseteq \frac{\varepsilon}{2} U_{m}$ and so (3.9) yields $U_{m} \cap P_{j_{0}}(X) \subseteq \frac{\varepsilon}{2} U_{m}+B$. Hence, for each $x \in U_{m}$ it follows from the previous inclusion, from the inclusion $P_{j_{0}}\left(U_{m}\right) \subseteq U_{m} \subseteq U_{n}$ and from (3.8) that

$$
\begin{aligned}
x & =\left(x-P_{j_{0}} x\right)+P_{j_{0}} x \in \frac{\varepsilon}{2} U_{n}+P_{j_{0}}\left(U_{m}\right) \\
& \subseteq \frac{\varepsilon}{2} U_{n}+\left(U_{m} \cap P_{j_{0}}(X)\right) \subseteq \frac{\varepsilon}{2} U_{n}+\frac{\varepsilon}{2} U_{m}+B \subseteq B+\varepsilon U_{n} .
\end{aligned}
$$

This establishes (3.7) with $L_{\varepsilon}:=B$ and completes the proof.

The following fact is an immediate consequence of Propositions 3.5 and 3.6.

Corollary 3.7. Let $X$ be a Fréchet space with basis $\left\{e_{i}\right\}_{i=1}^{\infty}$ and let $P^{(j)}$ be the projection defined via (2.4), for each $j \in \mathbb{N}$. Then $X$ is Schwartz if and only if $(R)-\lim _{j \rightarrow \infty} P^{(j)}=I$. 


\section{Power bounded operators on Fréchet-Schwartz spaces}

Let $X$ be a lcHs and $T \in \mathcal{L}(X)$, in which case we define $T_{[0]}:=I$ and

$$
T_{[k]}:=\frac{1}{k} \sum_{m=1}^{k} T^{m}, \quad k \in \mathbb{N} .
$$

The operator $T_{[k]}$ is the $k$-th Cesàro mean of $T$. Observe that

$$
(I-T) T_{[k]}=T_{[k]}(I-T)=\frac{1}{k}\left(T-T^{k+1}\right), \quad k \in \mathbb{N},
$$

and that

$$
\frac{1}{k} \cdot T^{k}=T_{[k]}-\frac{(k-1)}{k} T_{[k-1]}, \quad k \in \mathbb{N} .
$$

Suppose that $T_{[k]} \rightarrow P$ in $\mathcal{L}_{s}(X)$ as $k \rightarrow \infty$. According to (4.3) we have that $\tau_{s}-\lim _{k \rightarrow \infty} \frac{T^{k}}{k}=0$. Moreover, if $X$ is a Fréchet space, then $P$ is a projection satisfying $T P=P T=T$ with $\operatorname{Ker} P=\overline{(I-T)(X)}$ and $P(X)=\operatorname{Ker}(I-T)$. In addition,

$$
X=\operatorname{Ker}(I-T) \oplus \overline{(I-T)(X)},
$$

[1, Theorem 2.4], [27, Chap.VIII, §3, p.213]. Recall that $T \in \mathcal{L}(X)$ is power bounded if the sequence $\left\{T^{n}\right\}_{n=1}^{\infty}$ is equicontinuous in $\mathcal{L}(X)$ and that $T \in \mathcal{L}(X)$ is mean ergodic (resp. uniformly mean ergodic) if the sequence of Cesàro means $\left\{T_{[k]}\right\}_{k=1}^{\infty}$ is convergent in $\mathcal{L}_{s}(X)$ (resp. in $\mathcal{L}_{b}(X)$ ); see [1], [19], for more details. Finally, for $X$ a Fréchet space, an operator $T \in \mathcal{L}(X)$ is said to be rapidly mean ergodic, briefly $(R)$-mean ergodic, if the sequence $\left\{T_{[k]}\right\}_{k=1}^{\infty}$ is rapidly convergent in $\mathcal{L}(X)$. Rapid mean ergodicity always implies uniform mean ergodicity; see Remark 3.1.

Our purpose now is to investigate the connection between the power boundedness of an operator on a Fréchet-Schwartz space and the rapid convergence of its Cesàro means.

Proposition 4.1. Let $X$ be a Fréchet-Schwartz space. If $T \in \mathcal{L}(X)$ is power bounded, then $T$ is rapidly mean ergodic.

Proof. Since $X$ is Montel, [22, Remark 24.24], and $T$ is power bounded, we can apply [1, Theorem 2.4 and Proposition 2.8] to conclude that there is a projection $P \in \mathcal{L}(X)$ such that $T_{[k]} \rightarrow P$ in $\mathcal{L}_{b}(X)$ for $k \rightarrow \infty$. By Proposition 3.3 it follows that $(R)-\lim _{k \rightarrow \infty} T_{[k]}=P$.

Remark 4.2. Let $X$ be a Fréchet space with a basis. If $X$ is not Montel, then there exists a power bounded operator $T \in \mathcal{L}(X)$ such that $\left\{T_{[k]}\right\}_{k=1}^{\infty}$ does not converge in $\mathcal{L}_{b}(X)$, [1, Theorem 1.3]. Hence, $\left\{T_{[k]}\right\}_{k=1}^{\infty}$ cannot be rapidly convergent; see Remark 3.1.

The next result follows from a deep theorem of Bellenot, [4, Theorem 3.2].

Proposition 4.3. Let $X$ be a Fréchet space which is a closed subspace of a Fréchet space with an unconditional basis. If $X$ is not Schwartz, then there exist a closed subspace $Y$ of $X$ with an unconditional basis (say, $\left\{e_{i, j}\right\}_{i, j=1}^{\infty} \subseteq Y$ ), an increasing, fundamental sequence of seminorms $\left\{\|\cdot\|_{k}\right\}_{k=1}^{\infty}$ in $X$, and positive numbers $\left\{b_{k, i}: k \leq i, i \in \mathbb{N}\right\}$ satisfying $1<b_{k, i}\left\|e_{i, j}\right\|_{k}<2$ for each $k \leq i$ and $j \in \mathbb{N}$. Moreover, $Y$ is not Schwartz. 
Proof. We refer to [4, Theorem 3.2(II) and Corollary 3.4], together with the following comments.

In the notation of Definition 3.1 of [4], we use the partition $\left\{A_{i}\right\}_{i=1}^{\infty}$ of $\mathbb{N}$ given there (with each $A_{i}$ infinite) to write $\mathbb{N} \times \mathbb{N}=\cup_{i=1}^{\infty} A_{i}$ for the representation $A_{i}=\{(i, j): j \in \mathbb{N}\}$, for $i \in \mathbb{N}$. The obliquely normalized basic sequence $\left\{x_{n}\right\}_{n=1}^{\infty}$ in $\left[4\right.$, Definition 3.1] can then be written as $\left\{e_{i, j}\right\}_{i, j=1}^{\infty}$. Since the basic sequence $\left\{x_{n}\right\}_{n=1}^{\infty}$ is unconditional (cf. [4, Corollary 3.4]), the reordering $\left\{e_{i, j}\right\}_{i, j=1}^{\infty}$ of $\left\{x_{n}\right\}_{n=1}^{\infty}$ is permissible.

That $Y$ is not Schwartz is noted immediately prior to Theorem 3.2 in [4].

Let $X$ be a Fréchet space with an unconditional basis $\left\{e_{i}\right\}_{i=1}^{\infty}$ and corresponding dual basis $\left\{e_{i}^{\prime}\right\}_{i=1}^{\infty}$. For each finite subset $F \subseteq \mathbb{N}$, define $P_{F}: x \mapsto P_{F} x:=$ $\sum_{i \in F}\left\langle x, e_{i}^{\prime}\right\rangle e_{i}$, for $x \in X$ and, for each $j \in \mathbb{N}$, set $P^{(j)}:=P_{\{1, \ldots, j\}}$. Clearly, $P_{F} \in \mathcal{L}(X)$ is a finite-rank projection on $X$. Moreover, since the basis $\left\{e_{i}\right\}_{i=1}^{\infty}$ is unconditional, the family of operators $\left\{P_{F}: F \subseteq \mathbb{N}\right.$ finite $\}$ is a bounded subset of $\mathcal{L}_{s}(X)$ and hence, is equicontinuous (as $X$ is a Fréchet space). Furthermore, by $\left[20\right.$, Theorem 14.6.1] the set $\left\{P_{F} x: F \subseteq \mathbb{N}\right.$ finite $\}$ is precompact in $X$, for each $x \in X$.

Let $\left\{\|\cdot\|_{k}\right\}_{k=1}^{\infty}$ be a fundamental, increasing sequence of continuous seminorms for $X$. For fixed $k \in \mathbb{N}$, set

$$
q_{k}(x):=\sup \left\{\left\|P_{F} x\right\|_{k}: F \subseteq \mathbb{N} \text { finite }\right\}, \quad x \in X .
$$

The supremum is finite because $\left\{P_{F} x: F \subseteq \mathbb{N}\right.$ finite $\} \in \mathcal{B}(X)$ for each $x \in X$. Clearly, $q_{k}$ is a seminorm on $X$. Since $x=\lim _{j \rightarrow \infty} P^{(j)} x$ for all $x \in X$, it follows that $\|x\|_{k}=\lim _{j \rightarrow \infty}\left\|P^{(j)} x\right\|_{k} \leq q_{k}(x)$ for all $x \in X$. On the other hand, as $\left\{P_{F}: F \subseteq \mathbb{N}\right.$ finite $\}$ is equicontinuous, there exist $l(k)>k$ and $C_{k}>0$ such that

$$
\left\|P_{F} x\right\|_{k} \leq C_{k}\|x\|_{l(k)}, \quad x \in X, F \subseteq \mathbb{N} \text { finite, }
$$

and hence, $q_{k}(x) \leq C_{k}\|x\|_{l(k)}$, for $x \in X$. Since $k \in \mathbb{N}$ is arbitrary, we deduce that $\left\{q_{k}\right\}_{k=1}^{\infty}$ is also a fundamental, increasing sequence of seminorms in $X$. In particular, for each $i, k \in \mathbb{N}$, observe that $q_{k}\left(e_{i}\right)=\left\|e_{i}\right\|_{k}$ because $P_{F} e_{i}=0$ if $i \notin F$ and $P_{F} e_{i}=e_{i}$ if $i \in F$.

Lemma 4.4. Let $Y$ be a Fréchet space with an unconditional basis $\left\{e_{i}\right\}_{i=1}^{\infty}$ and corresponding dual basis $\left\{e_{i}^{\prime}\right\}_{i=1}^{\infty}$. Let $\left\{\lambda_{i}\right\}_{i=1}^{\infty}$ be a sequence of numbers satisfying $\left|\lambda_{i}\right| \leq 1$, for $i \in \mathbb{N}$. Then the diagonal operator $T_{\lambda}: Y \rightarrow Y$ given by

$$
T_{\lambda} x:=\sum_{i=1}^{\infty} \lambda_{i}\left\langle x, e_{i}^{\prime}\right\rangle e_{i}, \quad x \in Y,
$$

belongs to $\mathcal{L}(Y)$ and is power bounded.

Moreover, $\tau_{s}-\lim _{n \rightarrow \infty}\left(T_{\lambda}\right)^{n}=0$ whenever $\left|\lambda_{i}\right|<1$ for all $i \in \mathbb{N}$.

Proof. Let $\left\{\|\cdot\|_{k}\right\}_{k=1}^{\infty}$ be a fundamental, increasing sequence of seminorms on $Y$. By the comments immediately prior to the lemma (keeping the same notation), the seminorms $\left\{q_{k}\right\}_{k=1}^{\infty}$ given by (4.5) also form a fundamental, increasing sequence of seminorms on $Y$. Clearly,

$$
q_{k}\left(P_{F} x\right) \leq q_{k}(x), \quad x \in X, k \in \mathbb{N}, F \subseteq \mathbb{N} \text { finite. }
$$

For $x \in Y$, the series $\sum_{i=1}^{\infty}\left\langle x, e_{i}^{\prime}\right\rangle e_{i}$ converges unconditionally to $x$ in $Y$. So, by $\left[23, \S 4\right.$, Theorem], the series $\sum_{i=1}^{\infty} \mu_{i}\left\langle x, e_{i}^{\prime}\right\rangle e_{i}$ converges in $Y$ for all $\mu=\left(\mu_{i}\right)_{i} \in \ell^{\infty}$. 
Accordingly, the operator $T_{\lambda}: Y \rightarrow Y$ specified by (4.6) is well defined and linear. Moreover, as $T_{\lambda} x=\lim _{j \rightarrow \infty} \sum_{i=1}^{j} \lambda_{i}\left\langle x, e_{i}^{\prime}\right\rangle e_{i}$, for $x \in Y$, we can apply the BanachSteinhaus theorem to conclude that $T_{\lambda} \in \mathcal{L}(Y)$.

Fix $n \in \mathbb{N}$. Clearly, $\left|\lambda_{i}^{n}\right| \leq 1$ for all $i \in \mathbb{N}$. Hence, given $j \in \mathbb{N}$ and $k \in \mathbb{N}$, we can apply the inequality (I) on p.115 of [23] to obtain, for each $x \in Y$, via (4.7), that

$$
\begin{aligned}
q_{k}\left(\sum_{i=1}^{j} \lambda_{i}^{n}\left\langle x, e_{i}^{\prime}\right\rangle e_{i}\right) & \leq 4 \sup _{F \subseteq\{1, \ldots, j\}} q_{k}\left(\sum_{i \in F}\left\langle x, e_{i}^{\prime}\right\rangle e_{i}\right) \\
& =4 \sup _{F \subseteq\{1, \ldots, j\}} q_{k}\left(P_{F} x\right) \leq 4 q_{k}(x) .
\end{aligned}
$$

Since $\left(T_{\lambda}\right)^{n} x=\lim _{j \rightarrow \infty} \sum_{i=1}^{j} \lambda_{i}^{n}\left\langle x, e_{i}^{\prime}\right\rangle e_{i}$, for all $x \in Y$, it follows from (4.8), for every $k \in \mathbb{N}$, that

$$
q_{k}\left(\left(T_{\lambda}\right)^{n} x\right)=\lim _{j \rightarrow \infty} q_{k}\left(\sum_{i=1}^{j} \lambda_{i}^{n}\left\langle x, e_{i}^{\prime}\right\rangle e_{i}\right) \leq 4 q_{k}(x), \quad x \in Y .
$$

By the arbitrariness of $n \in \mathbb{N}$, it follows that $T_{\lambda}$ is power bounded.

Finally, assume that $\left|\lambda_{i}\right|<1$ for each $i \in \mathbb{N}$. This ensures that $\left(T_{\lambda}\right)^{n} e_{i}=$ $\lambda_{i}^{n} e_{i} \rightarrow 0$ in $Y$ for $n \rightarrow \infty$. Since $\left\{\left(T_{\lambda}\right)^{n}\right\}_{n=1}^{\infty}$ is equicontinuous and $\operatorname{span}\left\{e_{i}: i \in\right.$ $\mathbb{N}\}$ is dense in $Y$, it follows that $\left(T_{\lambda}\right)^{n} x \rightarrow 0$ in $Y$ as $n \rightarrow \infty$, for each $x \in Y$.

Proposition 4.5. Let $Y$ be a Fréchet space with a fundamental, increasing sequence of seminorms $\left\{\|\cdot\|_{k}\right\}_{k=1}^{\infty}$ and an unconditional basis $\left\{e_{i, j}\right\}_{i, j=1}^{\infty}$ such that there exist positive numbers $\left\{b_{k, i}: k \leq i, i \in \mathbb{N}\right\}$ satisfying $1<b_{k, i}\left\|e_{i, j}\right\|_{k}<2$ for each $k \leq i$ and $j \in \mathbb{N}$. Then there exists a power bounded operator $T \in \mathcal{L}(Y)$ such that $\tau_{s}-\lim _{n \rightarrow \infty} T^{n}=0$ but $T$ is not rapidly mean ergodic.

Proof. By assumption the basis $\left\{e_{i, j}\right\}_{i, j=1}^{\infty}$ is unconditional. In the notation of the discussion prior to Lemma 4.4, via (4.5) we have that $\left\{q_{k}\right\}_{k=1}^{\infty}$ is also a fundamental, increasing sequence of seminorms for $Y$ and $q_{k}\left(e_{i, j}\right)=\left\|e_{i, j}\right\|_{k}$ for each $k \in \mathbb{N}$ and $(i, j) \in \mathbb{N} \times \mathbb{N}$.

Set $\lambda:=\left(\lambda_{i j}\right)_{i, j \in \mathbb{N}}$ with $\lambda_{i j}:=1-2^{-j}$, for $i, j \in \mathbb{N}$. By Lemma 4.4 the diagonal operator $T \in \mathcal{L}(Y)$ given by

$$
T x:=\sum_{i, j=1}^{\infty} \lambda_{i j}\left\langle x, e_{i, j}^{\prime}\right\rangle e_{i, j}, \quad x \in Y,
$$

is power bounded and $\tau_{s}$ - $\lim _{n \rightarrow \infty} T^{n}=0$. It remains to show that the sequence $\left\{T_{[m]}\right\}_{m=1}^{\infty}$ is not rapidly convergent to 0 . For this, it suffices to show that the sequence $\left\{\sup _{x \in U_{k}} q_{1}\left(T_{[m]} x\right)\right\}_{m=1}^{\infty}$ fails to converge to 0 for each $k \in \mathbb{N}$. So, fix $k \in \mathbb{N}$. Then, for every $m, j \in \mathbb{N}$, we have

$$
\begin{aligned}
T_{[m]} e_{k, j} & =\frac{1}{m} \sum_{l=1}^{m} T^{l}\left(e_{k, j}\right)=\left(\frac{1}{m} \sum_{l=1}^{m}\left(\lambda_{k j}\right)^{l}\right) e_{k, j} \\
& =\left(\frac{1}{m} \lambda_{k j} \frac{1-\left(\lambda_{k j}\right)^{m}}{1-\lambda_{k j}}\right) e_{k, j} .
\end{aligned}
$$


For $m=2^{s}, j=s$, with $s \in \mathbb{N}$, it follows that

$$
\begin{aligned}
T_{\left[2^{s}\right]} e_{k, s} & =\left(\frac{1}{2^{s}}\left(1-2^{-s}\right) \frac{1-\left(1-2^{-s}\right)^{2^{s}}}{2^{s}}\right) e_{k, s} \\
& =\left(1-2^{-s}\right)\left[1-\left(1-2^{-s}\right)^{2^{s}}\right] e_{k, s}, \quad s \in \mathbb{N} .
\end{aligned}
$$

Since $q_{k}\left(e_{k, s} /\left\|e_{k, s}\right\|_{k}\right)=1$, the element $z_{k, s}:=e_{k, s} /\left\|e_{k, s}\right\|_{k} \in U_{k}$. Moreover, the sequence $\left\{1-\left(1-2^{-s}\right)^{2^{s}}\right\}_{s=1}^{\infty}$ converges to $\left(1-e^{-1}\right)$ for $s \rightarrow \infty$ and so, via (4.9), we obtain, for all $s$ large enough, that

$$
\begin{aligned}
& q_{1}\left(T_{\left[2^{s}\right]} z_{k, s}\right)=\left(1-2^{-s}\right)\left[1-\left(1-2^{-s}\right)^{2^{s}}\right] q_{1}\left(z_{k, s}\right) \geq \frac{1}{4}\left(1-\frac{1}{e}\right) \frac{q_{1}\left(e_{k, s}\right)}{\left\|e_{k, s}\right\|_{k}} \\
& =\frac{1}{4}\left(1-\frac{1}{e}\right) \frac{\left\|e_{k, s}\right\|_{1}}{\left\|e_{k, s}\right\|_{k}} .
\end{aligned}
$$

But, $\left\|e_{k, s}\right\|_{1}>1 / b_{1, k}$ and $\left\|e_{k, s}\right\|_{k}<2 / b_{k, k}$ for all $s \in \mathbb{N}$. So, from (4.10) it follows, for all $s \in \mathbb{N}$ large enough, that

$$
q_{1}\left(T_{\left[2^{s}\right]} z_{k, s}\right) \geq \frac{1}{4}\left(1-\frac{1}{e}\right) \frac{b_{k, k}}{2 b_{1, k}}
$$

Accordingly, $\left\{\sup _{x \in U_{k}} q_{1}\left(T_{[m]} x\right)\right\}_{m=1}^{\infty}$ cannot converge to 0 .

We can now establish one of the main results of this note.

Theorem 4.6. Let $X$ be a Fréchet space which is a closed subspace of a Fréchet space with an unconditional basis. Then $X$ is Schwartz if and only if every closed subspace $Y$ of $X$ has the property that every power bounded operator on $Y$ is rapidly mean ergodic.

Proof. Suppose that $X$ is Schwartz and $Y$ is any closed subspace of $X$. Then $Y$ is also Schwartz. Hence, every power bounded operator in $\mathcal{L}(Y)$ is rapidly mean ergodic; see Proposition 4.1.

Conversely, assume that $X$ is not Schwartz. By Proposition 4.3 there exists a closed subspace $Y$ of $X$ with an unconditional basis $\left\{e_{i, j}\right\}_{i, j=1}^{\infty}$ satisfying the assumptions of Proposition 4.5. So, by Proposition 4.5 there exists a power bounded operator $T \in \mathcal{L}(Y)$ which is not rapidly mean ergodic.

Remark 4.7. In [3] Bellenot exhibited Fréchet-Montel spaces which cannot be a closed subspace of any Fréchet space with an unconditional basis. Moreover, he proved that every Fréchet-Schwartz space is a closed subspace of some FréchetSchwartz space with an unconditional basis.

\section{Power bounded operators on Schwartz Köthe echelon spaces}

The aim of this section is to present a refinement of Theorem 4.6 when $X$ is a Köthe echelon space $\lambda_{p}(A)$, for $p \in[1, \infty) \cup\{0\}$. All members of this important and classical class of Fréchet spaces possess an unconditional basis.

A sequence $A=\left(a_{n}\right)_{n}$ of functions $a_{n}: I \rightarrow[0, \infty)$, with $I$ a non-void set, is called a Köthe matrix on $I$ if $0 \leq a_{n}(i) \leq a_{n+1}(i)$, for all $i \in I$ and $n \in \mathbb{N}$, and if 
for each $i \in I$ there is $n \in \mathbb{N}$ such that $a_{n}(i)>0$. To each $p \in[1, \infty)$ we associate the linear space

$$
\lambda_{p}(A, I):=\left\{x \in \mathbb{C}^{I}: q_{n}^{(p)}(x):=\left(\sum_{i \in I}\left|a_{n}(i) x_{i}\right|^{p}\right)^{1 / p}<\infty, \quad \forall n \in \mathbb{N}\right\} .
$$

We will also require the linear space

$$
\lambda_{\infty}(A, I):=\left\{x \in \mathbb{C}^{I}: q_{n}^{(\infty)}(x):=\sup _{i \in I} a_{n}(i)\left|x_{i}\right|<\infty, \forall n \in \mathbb{N}\right\}
$$

and its closed subspace (equipped with the relative topology)

$$
\lambda_{0}(A, I):=\left\{x \in \mathbb{C}^{I}: \lim _{i} a_{n}(i) x_{i}=0, \quad \forall n \in \mathbb{N}\right\} .
$$

The seminorms generating the topology of $\lambda_{0}(A, I)$ are, of course, the restrictions $q_{n}^{(0)}$ of $q_{n}^{(\infty)}$ to $\lambda_{0}(A, I)$, for $n \in \mathbb{N}$.

Elements $x \in \mathbb{C}^{I}$ are denoted by $x=\left(x_{i}\right)_{i}$. The spaces $\lambda_{p}(A, I)$, for $p \in[1, \infty]$, are called Köthe echelon spaces (of order $p$ ); they are all Fréchet spaces (separable if $I$ is countable and $p \neq \infty$ and reflexive if $p \notin\{0,1, \infty\}$ ) relative to the increasing sequence of seminorms $q_{1}^{(p)} \leq q_{2}^{(p)} \leq \ldots$. In case $I=\mathbb{N}$ or $I=\mathbb{N} \times \mathbb{N}$, we simply write $\lambda_{p}(A)$. In this case $\lambda_{p}(A)$, for $p \neq \infty$, has an unconditional basis. For the theory of such spaces we refer to [6], [7], [17], [22].

We begin with an example showing that in Proposition 3.6 it is not possible to remove the finite-dimensionality of the Schauder decomposition $\left\{P_{j}\right\}_{j=1}^{\infty}$, even if one replaces this condition with the requirement that each closed subspace $P_{j}(X)$, for $j \in \mathbb{N}$, is nuclear.

Example 5.1. Consider the Köthe matrix $A=\left(a_{n}\right)_{n}$ on $\mathbb{N} \times \mathbb{N}$ with entries

$$
a_{n}(i, j)= \begin{cases}(n j)^{n} & \text { if } i<n \\ n^{i} & \text { if } i \geq n\end{cases}
$$

Then the Köthe echelon space $\lambda_{1}(A)$ is Fréchet-Montel but not Fréchet-Schwartz; see [22, Example 27.21, p.338]. For each $k \in \mathbb{N}$, let $P_{k} \in \mathcal{L}\left(\lambda_{1}(A)\right)$ be given by $P_{k}\left(x_{i j}\right)_{i, j}:=\left(y_{i j}\right)_{i, j}$ where $y_{i j}:=x_{i j}$ if $i \leq k$ and $y_{i j}:=0$ if $i>k$, i.e., $P_{k}$ is the projection of $\lambda_{1}(A)$ onto its first $k$-rows. Then $\left\{P_{k}\right\}_{k=1}^{\infty}$ is a Schauder decomposition of $\lambda_{1}(A)$ but, surely not finite-dimensional.

The claim is that $P_{k}\left(\lambda_{1}(A)\right) \subseteq \lambda_{1}(A)$ is a nuclear Fréchet space for each $k \in \mathbb{N}$. To establish this it suffices to show that each sectional subspace

$$
Q_{s}\left(\lambda_{1}(A)\right):=\left\{x \in \lambda_{1}(A): x_{i j}=0 \text { if } i \neq s\right\}, \quad s \in \mathbb{N},
$$

is nuclear, because of the finite direct sum $P_{k}\left(\lambda_{1}(A)\right)=\oplus_{s=1}^{k} Q_{s}\left(\lambda_{1}(A)\right),[22$, Proposition 28.7].

So, fix $s \in \mathbb{N}$. If $n>s$, then $a_{n}(s, j)=(n j)^{n}$, for each $j \in \mathbb{N}$, and hence,

$$
\sum_{j=1}^{\infty} \frac{a_{n}(s, j)}{a_{n+2}(s, j)}=\sum_{j=1}^{\infty} \frac{n^{n}}{(n+2)^{n+2}} \frac{j^{n}}{j^{n+2}}=\frac{n^{n}}{(n+2)^{n+2}} \sum_{j=1}^{\infty} \frac{1}{j^{2}}<\infty .
$$

Recalling that our Köthe matrix $A=\left(a_{n}\right)_{n}$ is on $\mathbb{N} \times \mathbb{N}$ (rather than on $\mathbb{N}$ as in [22]) it follows from the Gröthendieck-Pietsch criterion, [22, Proposition 28.16], and (5.4) that $Q_{s}\left(\lambda_{1}(A)\right)$ is indeed nuclear. As already noted, the nuclearity of $P_{k}\left(\lambda_{1}(A)\right)$, for $k \in \mathbb{N}$, follows. 
It remains to prove that $P_{k} \stackrel{(R)}{\rightarrow} I$ in $\mathcal{L}\left(\lambda_{1}(A)\right)$ as $k \rightarrow \infty$. To see this, recall that the $n$-th seminorm $q_{n}:=q_{n}^{(1)}$ of $\lambda_{1}(A)$, for $n \in \mathbb{N}$, is given by $q_{n}(x):=$ $\sum_{i, j=1}^{\infty} a_{n}(i, j)\left|x_{i j}\right|$, for $x \in \lambda_{1}(A)$; see (5.1). Fix any $n \in \mathbb{N}$ and set $m:=2 n$. Then, for any $k \geq m$, we have via (5.3) that

$$
\begin{aligned}
q_{n}\left(\left(I-P_{k}\right) x\right) & =\sum_{i \geq k+1} \sum_{j=1}^{\infty} a_{n}(i, j)\left|x_{i j}\right|=\sum_{i \geq k+1} \sum_{j=1}^{\infty} n^{i}\left|x_{i j}\right| \\
& =\sum_{i \geq k+1} 2^{-i} \sum_{j=1}^{\infty}(2 n)^{i}\left|x_{i j}\right| \leq\left(\sum_{i \geq k+1} 2^{-i}\right) q_{m}(x),
\end{aligned}
$$

because $i \geq k \geq 2 n$ yields

$$
\sum_{j=1}^{\infty}(2 n)^{i}\left|x_{i j}\right|=\sum_{j=1}^{\infty} a_{2 n}(i, j)\left|x_{i j}\right| \leq \sum_{i, j=1}^{\infty} a_{2 n}(i, j)\left|x_{i j}\right|=q_{m}(x) .
$$

It is then clear from (5.5) that

$$
\sup _{x \in U_{m}} q_{n}\left(\left(I-P_{k}\right) x\right) \leq \sum_{i \geq k+1} 2^{-i}=2^{-k}, \quad k \geq m,
$$

which implies that $\lim _{k \rightarrow \infty} \sup _{x \in U_{m}} q_{n}\left(\left(I-P_{k}\right) x\right)=0$. Since $n$ is arbitrary, this means precisely that $P_{k} \stackrel{(R)}{\rightarrow} I$ in $\mathcal{L}\left(\lambda_{1}(A)\right)$ as $k \rightarrow \infty$.

Our purpose is to characterize those Köthe echelon spaces which are Schwartz, in terms of the behaviour of the Cesàro means of power bounded operators defined on them. This is obtained in Theorem 5.6 below, a result which improves Theorem 4.6 for the case of Köthe echelon spaces. For characterizing the property of being Schwartz, Theorem 4.6 also provides a version of analogous results characterizing Montel and reflexive Fréchet spaces; see [1, Proposition 5.1 and Theorem 5.4].

We first require the following result, which is implicit in [26, pp.223-224]. We include a proof for the sake of completeness.

Lemma 5.2. Let $A=\left(a_{n}\right)_{n \in \mathbb{N}}$ be a Köthe matrix on $\mathbb{N}$ which satisfies the following two conditions.

(M) For each $n \in \mathbb{N}$ and each infinite subset $H \subseteq \mathbb{N}$ there exists $m>n$ such that $\inf _{i \in H} \frac{a_{n}(i)}{a_{m}(i)}=0$.

(not-S) There exists $n_{0} \in \mathbb{N}$ such that $\frac{a_{1}}{a_{m}} \notin c_{0}$ for every $m>n_{0}$.

Then there exist an infinite family $\left\{I_{i}\right\}_{i=1}^{\infty}$ of pairwise disjoint, infinite subsets of $\mathbb{N}$, with each $I_{i}$ represented as $I_{i}=\{(i, j): j \in \mathbb{N}\}$, and an increasing sequence $\left\{m_{j}\right\}_{j=1}^{\infty} \subseteq \mathbb{N}$ beginning with $m_{1}=2$ satisfying:

(1) for each $i>2$ there exists $\varepsilon_{i}>0$ such that $a_{1}(i, j)>\varepsilon_{i} a_{m_{i}}(i, j)$ for all $(i, j) \in I_{i}$ with $j \in \mathbb{N}$, and

(2) for each $i \in \mathbb{N}$ we have $\lim _{j \rightarrow \infty} \frac{a_{m_{i}}(i, j)}{a_{m_{i+1}}(i, j)}=0$.

Proof. Choose $n_{0}$ according to (not-S). Deleting finitely many $n$ 's, if necessary, we may assume that $n_{0}=1$ and that $a_{n}>0$ on $\mathbb{N}$ for all $n \in \mathbb{N}$. Since $\frac{a_{1}}{a_{2}} \notin c_{0}$, there exist $\varepsilon_{1}>0$ and an increasing sequence $J_{1}=\left\{l_{s}^{1}\right\}_{s=1}^{\infty} \subseteq \mathbb{N}$ such that $\frac{a_{1}\left(l_{s}^{1}\right)}{a_{2}\left(l_{s}^{1}\right)}>\varepsilon_{1}$ 
for all $s \in \mathbb{N}$. By condition $(\mathrm{M})$ there exist $m_{2}>m_{1}:=2$ and an infinite subset $I_{1}$ of $J_{1}$, which can be represented as $I_{1}=\{(1, j): j \in \mathbb{N}\}$, such that $\lim _{j \rightarrow \infty} \frac{a_{2}(1, j)}{a_{m_{2}}(1, j)}=0$. Since $0<a_{1} \leq a_{2}$ on $\mathbb{N}$, we have $0<\frac{a_{1}}{a_{m_{2}}} \leq \frac{a_{2}}{a_{m_{2}}}$ on $\mathbb{N}$ and hence, also $\lim _{j \rightarrow \infty} \frac{a_{1}(1, j)}{a_{m_{2}}(1, j)}=0$. By (not-S), also $\frac{a_{1}}{a_{m_{2}}} \notin c_{0}$. So, there exist $\varepsilon_{2}>0$ and an increasing sequence $J_{2}=\left\{l_{s}^{2}\right\}_{s=1}^{\infty} \subseteq \mathbb{N} \backslash I_{1}$ such that $\frac{a_{1}\left(l_{s}^{2}\right)}{a_{m_{2}}\left(l_{s}^{2}\right)}>\varepsilon_{2}$ for all $s \in \mathbb{N}$. Then, by condition (M) there exist $m_{3}>m_{2}$ and an infinite subset $I_{2}$ of $J_{2}$, which can be represented as $I_{2}=\{(2, j): j \in \mathbb{N}\}$, such that $\lim _{j \rightarrow \infty} \frac{a_{m_{2}}(2, j)}{a_{m_{3}}(2, j)}=0$. Note that $0<\frac{a_{1}}{a_{m_{3}}} \leq \frac{a_{1}}{a_{m_{2}}}$ on $I_{1}$ (as $a_{m_{2}} \leq a_{m_{3}}$ ) and $0<\frac{a_{1}}{a_{m_{3}}} \leq \frac{a_{m_{2}}}{a_{m_{3}}}$ on $I_{2}$ (as $\left.a_{1} \leq a_{m_{2}}\right)$. Since $I_{1} \cap I_{2}=\emptyset$, with $\frac{a_{1}}{a_{m_{2}}} \rightarrow 0$ on $I_{1}$ and $\frac{a_{m_{2}}}{a_{m_{3}}} \rightarrow 0$ on $I_{2}$, it follows that $\lim _{k \rightarrow \infty}, k \in\left(I_{1} \cup I_{2}\right) \frac{a_{1}(k)}{a_{m_{3}}(k)}=0$. Again by (not-S), also $\frac{a_{1}}{a_{m_{3}}} \notin c_{0}$. So, there exist $\varepsilon_{3}>0$ and an increasing sequence $J_{3}=\left\{l_{s}^{3}\right\}_{s=1}^{\infty} \subseteq \mathbb{N} \backslash\left(I_{1} \cup I_{2}\right)$ such that $\frac{a_{1}\left(l_{s}^{3}\right)}{a_{m_{3}}\left(l_{s}^{3}\right)}>\varepsilon_{3}$ for all $s \in \mathbb{N}$. Then, by condition $(\mathrm{M})$ there exist $m_{4}>m_{3}$ and an infinite subset $I_{3}$ of $J_{3}$, which can be represented as $I_{3}=\{(3, j): j \in \mathbb{N}\}$, such that $\lim _{j \rightarrow \infty} \frac{a_{m_{3}}(3, j)}{a_{m_{4}}(3, j)}=0$.

Proceeding by induction, the proof follows.

Let us return to the spaces $\lambda_{p}(A)$ with $p \in[1, \infty) \cup\{0\}$.

Corollary 5.3. Let $A=\left(a_{n}\right)_{n \in \mathbb{N}}$ be a Köthe matrix on $\mathbb{N}$. If the Köthe echelon space $\lambda_{p}(A)$, with $p \in[1, \infty) \cup\{0\}$, is Montel but not Schwartz, then there exists a sectional subspace $\lambda_{p}(A, I)$ of $\lambda_{p}(A)$, with $I=\{(i, j): i, j \in \mathbb{N}\}$, possessing the following two properties.

(1) For each $i \geq 2$ there exists $\varepsilon_{i}>0$ such that $a_{1}(i, j)>\varepsilon_{i} a_{i}(i, j)$ for all $j \in \mathbb{N}$.

(2) For each $i \in \mathbb{N}$ we have $\lim _{j \rightarrow \infty} \frac{a_{i}(i, j)}{a_{i+1}(i, j)}=0$.

Moreover, $\lambda_{p}(A, I)$ is Montel.

Proof. Since $\lambda_{p}(A)$ is Montel but not Schwartz, the Köthe matrix $A$ satisfies the condition (M), [22, Theorem 27.9], and the condition (not-S), [22, Proposition 27.10], given in Lemma 5.2. So, by Lemma 5.2 there exist an infinite family $\left\{I_{i}\right\}_{i=1}^{\infty}$ of pairwise disjoint infinite subsets of $\mathbb{N}$, where $I_{i}$ can be represented as $I_{i}=\{(i, j): j \in \mathbb{N}\}$, and an increasing sequence $\left\{m_{j}\right\}_{j=1}^{\infty} \subseteq \mathbb{N}$ beginning with $m_{1}=2$ such that both conditions (1) and (2) of Lemma 5.2 are satisfied.

Set $I:=\cup_{i=1}^{\infty} I_{i}=\{(i, j): i, j \in \mathbb{N}\}$ and pass to the subsequence $\left\{a_{m_{j}}\right\}_{j=1}^{\infty}$ of $\left\{a_{n}\right\}_{n=1}^{\infty}$. Then the sectional subspace $\lambda_{p}(A, I)$ of $\lambda_{p}(A)$ satisfies conditions (1) and (2) in the statement of this corollary.

Lemma 5.4. Let $p \in[1, \infty) \cup\{0\}$ and $A=\left(a_{n}\right)_{n \in \mathbb{N}}$ be a Köthe matrix on $\mathbb{N} \times \mathbb{N}$ such that the Köthe echelon space $\lambda_{p}(A)$ is Montel. If A satisfies

$$
\forall n \geq 2 \exists \varepsilon_{n}>0 \forall j \in \mathbb{N} \quad a_{1}(n, j) \geq \varepsilon_{n} a_{n}(n, j),
$$

then there exists a power bounded operator $T \in \mathcal{L}\left(\lambda_{p}(A)\right)$ satisfying $\tau_{b}-\lim _{n \rightarrow \infty} T^{n}=$ 0 but $\left\{T_{[k]}\right\}_{k=1}^{\infty}$ is not rapidly convergent to 0 .

Proof. Set $\lambda_{j}:=1-2^{-j}$, for $j \in \mathbb{N}$, and define $T x:=\left(\lambda_{j} x_{i j}\right)_{i, j}$, for $x=\left(x_{i j}\right)_{i, j} \in$ $\lambda_{p}(A)$. Then $T: x \mapsto T x$ belongs to $\mathcal{L}\left(\lambda_{p}(A)\right)$ and is power bounded. Indeed, 
given $n \in \mathbb{N}$, we have for each $m \in \mathbb{N}$ and $x \in \lambda_{p}(A)$, that $q_{m}^{(p)}\left(T^{n} x\right) \leq q_{m}^{(p)}(x)$, as $0<\left(\lambda_{j}\right)^{n}<1$ for all $j \in \mathbb{N}$; see (5.1) and (5.2). So, $\left\{T^{n}\right\}_{n=1}^{\infty}$ is equicontinuous.

For each $i, j \in \mathbb{N}$, set $e_{i, j}:=\left(\delta_{h i} \delta_{k j}\right)_{h, k}$, where $\delta_{r s}=0$ if $r \neq s$ and $\delta_{r s}=1$ if $r=s$. Then, for each $n \in \mathbb{N}$ and $(i, j) \in \mathbb{N} \times \mathbb{N}$, we have $T^{n} e_{i, j}=\left(\lambda_{j}\right)^{n} e_{i, j}$. In particular, $\lim _{n \rightarrow \infty} T^{n} e_{i, j}=0$ in $\lambda_{p}(A)$ for each $i, j \in \mathbb{N}$. Since $\left\{T^{n}\right\}_{n=1}^{\infty}$ is equicontinuous and $\operatorname{span}\left\{e_{i, j}: i, j \in \mathbb{N}\right\}$ is dense in $\lambda_{p}(A)$, it follows that $\tau_{s^{-}}$ $\lim _{n \rightarrow \infty} T^{n}=0$. Since $\lambda_{p}(A)$ is Montel, we also have that $\tau_{b}-\lim _{n \rightarrow \infty} T^{n}=0$. Accordingly, also the arthmetic means $\left\{T_{[k]}\right\}_{k=1}^{\infty}$ of $\left\{T^{n}\right\}_{n=1}^{\infty}$ converge to 0 in $\mathcal{L}_{b}\left(\lambda_{p}(A)\right)$, i.e., $T$ is uniformly mean ergodic with limit projection $P=0$.

It remains to show that $\left\{T_{[k]}\right\}_{k=1}^{\infty}$ is not rapidly convergent to 0 in $\mathcal{L}\left(\lambda_{p}(A)\right)$. For this it suffices to show that the sequence $\left\{\sup _{x \in U_{m}} q_{1}^{(p)}\left(T_{[k]} x\right)\right\}_{k=1}^{\infty}$ fails to converge to 0 for each $m \geq 2$, where $U_{m}:=\left\{x \in \lambda_{p}(A): q_{m}^{(p)}(x) \leq 1\right\}$.

So, fix any $m \in \mathbb{N}$ with $m \geq 2$. For each $j, k \in \mathbb{N}$, we have

$$
\begin{aligned}
T_{[k]} e_{m, j} & =\frac{1}{k} \sum_{l=1}^{k} T^{l}\left(e_{m, j}\right)=\left(\frac{1}{k} \sum_{l=1}^{k} \lambda_{j}^{l}\right) e_{m, j} \\
& =\left(\frac{1}{k} \lambda_{j} \frac{1-\lambda_{j}^{k}}{1-\lambda_{j}}\right) e_{m, j} .
\end{aligned}
$$

If we take $k:=2^{s}$ and $j:=s$ for each $s \in \mathbb{N}$, then from (5.7) it follows that

$$
\begin{aligned}
T_{\left[2^{s}\right]} e_{m, s} & =\left[\frac{1}{2^{s}}\left(1-2^{-s}\right) \frac{1-\left(1-2^{-s}\right)^{2^{s}}}{2^{-s}}\right] e_{m, s} \\
& =\left(1-2^{-s}\right)\left[1-\left(1-2^{-s}\right)^{2^{s}}\right] e_{m, s} .
\end{aligned}
$$

Note that $q_{m}^{(p)}\left(\frac{e_{m, s}}{a_{m}(m, s)}\right)=1$ and so $z_{m, s}:=\frac{e_{m, s}}{a_{m}(m, s)} \in U_{m}$, for every $s \in \mathbb{N}$. Since the sequence $\left\{\left[1-\left(1-2^{-s}\right)^{2^{s}}\right]\right\}_{s=1}^{\infty}$ converges to $\left(1-e^{-1}\right)$ for $s \rightarrow \infty$, it follows via (5.8) and (5.6) that, for all $s \in \mathbb{N}$ large enough, we have

$$
\begin{aligned}
q_{1}^{(p)}\left(T_{\left[2^{s}\right]} z_{m, s}\right) & =\frac{1}{a_{m}(m, s)}\left(1-2^{-s}\right)\left[1-\left(1-2^{-s}\right)^{2^{s}}\right] q_{1}^{(p)}\left(e_{m, s}\right) \\
& \geq \frac{1}{4}\left(1-e^{-1}\right) \frac{a_{1}(m, s)}{a_{m}(m, s)} \geq \frac{1}{4}\left(1-e^{-1}\right) \varepsilon_{m} .
\end{aligned}
$$

This shows that the sequence $\left\{\sup _{x \in U_{m}} q_{1}^{(p)}\left(T_{[k]} x\right)\right\}_{k=1}^{\infty}$ cannot converge to 0 . Since $m \geq 2$ is arbitrary, the proof is complete.

Remark 5.5. Corollary 5.3 ensures that there exist Köthe matrices $A$ which satisfy the assumptions required in Lemma 5.4. For explicit examples, see [17, Ch. 31, §5], [22, Example 27.21].

Theorem 5.6. Let $p \in[1, \infty) \cup\{0\}$ and $A=\left(a_{n}\right)_{n \in \mathbb{N}}$ be a Köthe matrix on $\mathbb{N}$. Then the Köthe echelon space $\lambda_{p}(A)$ is Schwartz if and only if every power bounded operator on $\lambda_{p}(A)$ is rapidly mean ergodic.

Proof. If $\lambda_{p}(A)$ is Schwartz, then the desired conclusion follows from Proposition 4.1.

Conversely, suppose that $\lambda_{p}(A)$ is not Schwartz. 
Case (I). $\lambda_{p}(A)$ is not Montel. Then it follows from [1, Propositions 2.9 and 2.13] that there exists a power bounded operator $T \in \mathcal{L}\left(\lambda_{p}(A)\right)$ which is not uniformly mean ergodic and hence, by Remark 3.1, also not rapidly mean ergodic.

Case (II). $\lambda_{p}(A)$ is Montel. Then, by Corollary 5.3, there exists a sectional subspace $\lambda_{p}(A, I)$ of $\lambda_{p}(A)$, with $I=\mathbb{N} \times \mathbb{N}$, satisfying condition (5.6). So, by Lemma 5.4 there exists $T \in \mathcal{L}\left(\lambda_{p}(A, I)\right)$ which is power bounded, satisfies $\tau_{b^{-}} \lim _{n \rightarrow \infty} T^{n}=0$ but, $T$ is not rapidly mean ergodic.

Since $\lambda_{p}(A, I)$ is a sectional (hence, complemented) subspace of $\lambda_{p}(A)$, using the operator $T$ it is routine to construct $S \in \mathcal{L}_{b}\left(\lambda_{p}(A)\right)$ which is power bounded but not rapidly mean ergodic.

We conclude with some comments regarding the spaces $\lambda_{\infty}(A)$. First, the canonical unit vectors $\left\{e_{i}\right\}_{i=1}^{\infty}$ form an unconditional basis of $\lambda_{\infty}(A)$ if and only if $\lambda_{\infty}(A)$ is Montel if and only if $\lambda_{\infty}(A)=\lambda_{0}(A)$, [11, Proposition 2.3]. Moreover, there exist Köthe matrices $A$ such that $\lambda_{\infty}(A)$ is Montel but not Schwartz, [22, Example 27.21]. In this case, the projections $\left\{P^{(j)}\right\}_{j=1}^{\infty}$ defined via (2.4) form a finite-dimensional, unconditional Schauder decomposition of $\lambda_{\infty}(A)$ with $\tau_{b^{-}}$ $\lim _{j \rightarrow \infty} P^{(j)}=I$ (as $\lambda_{\infty}(A)$ is Montel) but, $\left\{P^{(j)}\right\}_{j=1}^{\infty}$ is not rapidly convergent to $I$ in $\mathcal{L}\left(\lambda_{\infty}\right)(A)$; see Corollary 3.7. It is also known that there exist Köthe matrices $A$ such that $\lambda_{\infty}(A)$ is not Montel, non-normable and satisfies the density condition, [11, pp.90-91]. Then $\left\{e_{i}\right\}_{i=1}^{\infty}$ fails to be an unconditional basis of $\lambda_{\infty}(A)$. Nevertheless, for such $A$, the space $\lambda_{\infty}(A)$ admits a non-trivial, unconditional Schauder decomposition, [11, Proposition 4.4].

Even though $\left\{e_{i}\right\}_{i=1}^{\infty}$ may not be a basis for $\lambda_{\infty}(A)$, in general, we can always define a linear functional $e_{i}^{\prime}: \lambda_{\infty}(A) \rightarrow \mathbb{C}$ via

$$
\left\langle x, e_{i}^{\prime}\right\rangle:=x_{i}, \quad x=\left(x_{j}\right)_{j} \in \lambda_{\infty}(A),
$$

for each $i \in \mathbb{N}$. For a fixed $n \in \mathbb{N}$, we have for each $i \in \mathbb{N}$, that

$$
\left|\left\langle x, e_{i}^{\prime}\right\rangle\right| \leq\left\{\begin{array}{lll}
q_{n}^{(\infty)}(x) & \text { if } a_{n}(i)=0, & x \in \lambda_{\infty}(A) \\
\left(a_{n}(i)\right)^{-1} q_{n}^{(\infty)}(x) & \text { if } a_{n}(i)>0, & x \in \lambda_{\infty}(A)
\end{array}\right.
$$

from which it is clear that $e_{i}^{\prime}$ is continuous. Moreover, the finite-rank projections $\left\{P^{(j)}\right\}_{j=1}^{\infty} \subseteq \mathcal{L}\left(\lambda_{\infty}(A)\right)$ as given by (2.4) are then well defined and satisfy both (S1), (S3) in the definition of a Schauder decomposition. In relation to the next result, see also [22, Proposition 27.10].

Proposition 5.7. The Köthe echelon space $\lambda_{\infty}(A)$ is Schwartz if and only if $(R)-\lim _{j \rightarrow \infty} P^{(j)}=I$.

Proof. If $\lambda_{\infty}(A)$ is Schwartz, then it is also Montel. By the comments prior to the proposition we have $\lambda_{\infty}(A)=\lambda_{0}(A)$ with $\left\{e_{i}\right\}_{i=1}^{\infty}$ an unconditional basis. In particular, $\left\{P^{(j)}\right\}_{j=1}^{\infty}$ is then a Schauder decomposition of $\lambda_{\infty}(A)$ and so $(R)$ $\lim _{j \rightarrow \infty} P^{(j)}=I$ in $\mathcal{L}\left(\lambda_{\infty}(A)\right)$; see Proposition 3.5.

Conversely, suppose that $(R)-\lim _{j \rightarrow \infty} P^{(j)}=I$ in $\mathcal{L}\left(\lambda_{\infty}(A)\right)$. By Remark 3.1 also $\tau_{s^{-}} \lim _{j \rightarrow \infty} P^{(j)}=I$ and so $\left\{P^{(j)}\right\}_{j=1}^{\infty}$ is a (finite-dimensional) Schauder decomposition of $\lambda_{\infty}(A)$. Then Proposition 3.6 implies that $\lambda_{\infty}(A)$ is Schwartz.

We conclude with the following analogue of Theorem 5.6. 
Proposition 5.8. The Köthe echelon space $\lambda_{\infty}(A)$ is Schwartz if and only if every power bounded operator on $\lambda_{\infty}(A)$ is rapidly mean ergodic.

Proof. If $\lambda_{\infty}(A)$ is Schwartz, the desired conclusion follows from Proposition 4.1. Conversely, suppose that $\lambda_{\infty}(A)$ is not Schwartz.

Case $(\mathrm{I}) . \lambda_{\infty}(A)$ is not Montel. By the equivalence $(1) \Leftrightarrow(6)$ in $[22$, Theorem 27.9], with $p=\infty$, there is an infinite set $J \subseteq \mathbb{N}$, a constant $K>0$ and $n \in \mathbb{N}$ such that $a_{m}(i) \leq K a_{n}(i)$ for all $i \in J$ and $m \geq n$. Since the sequence $\left\{a_{k}\right\}_{k=1}^{\infty}$ is increasing (pointwise on $\mathbb{N}$ ), we also have $a_{n}(i) \leq a_{m}(i)$ for all $i \in J$ and $m \geq n$. So, the complemented, sectional subspace $\lambda_{\infty}(A, J)$ is isomorphic to $\ell^{\infty}\left(a_{n} \mid J\right) \simeq \ell^{\infty}$. The operator $S \in \mathcal{L}\left(\ell^{\infty}\right)$ defined by $S x:=\left(x_{2}, x_{3}, x_{4}, \ldots\right)$, for $x=\left(x_{i}\right)_{i} \in \ell^{\infty}$, is power bounded but not uniformly mean ergodic. By Remark 3.1, $S$ is not rapidly mean ergodic. Using $S$ it is routine to construct $T \in \mathcal{L}\left(\lambda_{\infty}(A)\right)$ which is power bounded but not rapidly mean ergodic.

Case (II). $\lambda_{\infty}(A)$ is Montel. Then $\lambda_{\infty}(A)=\lambda_{0}(A)$ with $\lambda_{0}(A)$ Montel. By Case (II) in the proof of Theorem 5.6 (with $p=0$ ) there exists a power bounded operator in $\mathcal{L}\left(\lambda_{0}(A)\right)=\mathcal{L}\left(\lambda_{\infty}(A)\right)$ which is not rapidly mean ergodic.

Acknowledgements. The research of the first two authors was partially supported by the projects MTM2010-15200 and GVA Prometeo II/2013/013 (Spain). The second author gratefully acknowledges the support of the Alexander von Humboldt Foundation.

\section{REFERENCES}

[1] A.A. Albanese, J. Bonet, W.J. Ricker, Mean ergodic operators in Fréchet spaces. Ann. Acad. Sci. Fenn. Math. 34 (2009), 401-436.

[2] A.A. Albanese, J. Bonet, W.J. Ricker, On mean ergodic operators. In: Vector Measures, Integration and Related Topics, G.P. Curbera et. al. (Eds), Operator Theory: Advances and Applications 201, Birkhäuser Verlag, Basel, 2010, pp. 1-20.

[3] S.F. Bellenot, Each Schwartz-Fréchet space is a subspace of a Schwartz-Fréchet space with an unconditional basis. Compositio Math. 42 (1980/81), 273-278.

[4] S.F. Bellenot, Basic sequences in non-Schwartz Fréchet spaces. Trans. Amer. Math. Soc. 258 (1980), 199-216.

[5] A. Benndorf, On the relation of the bounded approximation property and a finitedimensional decomposition in nuclear Fréchet spaces. Studia Math. 75 (1983), 103-119.

[6] K.D. Bierstedt, J. Bonet, Some aspects of the modern theory of Fréchet spaces. Rev. R. Acad. Cienc. Exactas Fis. Nat. Ser. A. Math. RACSAM 97 (2003), 159-188.

[7] K.D. Bierstedt, R.G. Meise, W.H. Summers, Köthe sets and Köthe sequence spaces. In: "Functional Analysis, Holomorphy and Approximation Theory" (Rio de Janeiro, 1980), North Holland Math. Studies 71 (1982), pp. 27-91.

[8] J. Bonet, A question of Valdivia on quasinormable Fréchet spaces. Canad. Math. Bull. 34 (1991), 301-304.

[9] J. Bonet, M. Lindström, M. Valdivia, Two theorems of Josefson-Nissenzweig type for Fréchet spaces. Proc. Amer. Math. Soc. 117 (1993), 363-364.

[10] J. Bonet, B. de Pagter, W.J. Ricker, Mean ergodic operators and reflexive Fréchet lattices. Proc. Roc. Soc. Edinburgh 141A (2011), 897-920.

[11] J. Bonet, W.J. Ricker, Schauder decompositions and the Grothendieck and Dunford-Pettis properties in Köthe echelon spaces of infinite order. Positivity 11 (2007), 77-93.

[12] J.C. Díaz, On non-primary Fréchet Schwartz spaces. Studia Math. 126 (1997), 291-307.

[13] E. Yu. Emel'yanov, Banach lattices on which every power bounded operator is mean ergodic. Positivity 1 (1997), 291-296. 
[14] V.P. Fonf, M. Lin, P. Wojtaszczyk, Ergodic characterizations of reflexivity in Banach spaces. J. Funct. Anal. 187 (2001), 146-162.

[15] J. Horváth, Topological Vector Spaces and Distributions. Vol. I, Addison-Wesley Publishing Co., Reading, Mass.-London-Don Mills, Ont. 1966.

[16] N.J. Kalton, Schauder decompositions in locally convex spaces. Proc. Camb. Phil. Soc. 68 (1970), 377- 392.

[17] G. Köthe, Topological Vector Spaces I. 2nd Rev. Ed., Springer Verlag, Berlin-HeidelbergNew York, 1983.

[18] G. Köthe, Topological Vector Spaces II. Springer Verlag, Berlin-Heidelberg-New York, 1979.

[19] U. Krengel, Ergodic Theorems. de Gruyter Studies in Mathematics, 6. Walter de Gruyter Co., Berlin, 1985.

[20] H. Jarchow, Locally Convex Spaces. B.G. Teubner, Stuttgart, 1981.

[21] M. Lindström, T. Schlumprecht, A Josefson-Nissenzweig theorem for Fréchet spaces. Bull. London Math. Soc. 25 (1993), 55-58.

[22] R. Meise, D. Vogt, Introduction to Functional Analysis. Oxford Graduate Texts in Mathematics, 2. The Clarendon Press. Oxford University Press, New York, 1997.

[23] C.W. McArthur, J.R. Retherford, Some applications of an inequality in locally convex spaces. Trans. Amer. Math. Soc. 137 (1969), 115-123.

[24] K. Piszczek, Quasi-reflexive Fréchet spaces and mean ergodicity. J. Math. Anal. Appl. 361 (2010), 224-233.

[25] K. Piszczek, Barrelled spaces and mean ergodicity. Rev. R. Acad. Cienc. Exactas Fis. Nat. Ser. A. Math. RACSAM 104 (2010), 5-11.

[26] M. Valdivia, Topics in Locally Convex Spaces. North-Holland Mathematics Studies, 67, North-Holland Publishing Co., Amsterdam-New York, 1982.

[27] K. Yosida, Functional Analysis. Sixth Edition, Springer-Verlag, Berlin, 1980.

Angela A. Albanese, Dipartimento di Matematica e Fisica "E. De Giorgi", UniVersità del Salento- C.P.193, I-73100 LeCCe, Italy

E-mail address: angela.albanese@unisalento.it

José Bonet, Instituto Universitario de Matemática Pura y Aplicada iUmpa, Universidad Politécnica de Valencia, E-46071 Valencia, Spain

E-mail address: jbonet@mat.upv.es

Werner J. Ricker, Math.-Geogr. Fakultät, Katholische Universität EichstättIngolstadt, D-85072 Eichstätt, Germany

E-mail address: werner.ricker@ku-eichstaett.de 